\title{
Meta-Analysis
}

\section{$\Theta$ Effects of Intravenous and Oral Magnesium on Reducing Migraine: A Meta-analysis of Randomized Controlled Trials}

Hsiao-Yean Chiu RN, PhD ${ }^{1}$, Tu-Hsueh Yeh MD, PhD², Yin-Cheng Huang MD, PhD ${ }^{3,4}$, and Pin-Yuan Chen MD, $\mathrm{PhD}^{3,4}$

From: ${ }^{1}$ School of Nursing College of Nursing, Taipei Medical University, Taipei,

Taiwan; ${ }^{2}$ Department of Neurology, Chang Gung Memorial Hospital at Linkou, Taoyuan, Taiwan; ${ }^{3}$ Department of Neurosurgery, Chang Gung Memorial Hospital at Linkou,

Taoyuan, Taiwan; ${ }^{4}$ College of Medicine, Chang Gung University, Taoyuan, Taiwan

Address Correspondence: Pin-Yuan Chen, MD, PhD Department of Neurosurgery Chang Gung Memoria Hospital No.5, Fusing St. Gueishan Township, Taoyuan County 33305 Taiwan (R.O.C.)

E-mail: pinyuanc@gmail.com

Conflicts of Interest and Source of Funding: The authors declare no potential conflicts of interest with respect to the authorship and/or publication of this article. This meta-analysis was supported by a grant from Taipei Medical University, Taiwan (No. TMU1O3-AE1-B11).

Manuscript received: 04-07-2015

Revised manuscript received: 05-31-2015 Accepted for publication: 07-02-2015

Free full manuscript: www.painphysicianjournal.com
Background: Migraine attack has been associated with magnesium deficiency. Previous studies investigating the effect of intravenous and oral magnesium on acute migraine attacks and the prevention of migraine have produced equivocal findings.

Objective: To evaluate the effects of intravenous magnesium on acute migraine attacks and oral magnesium supplements on migraine prophylaxis.

Study Design: A meta-analysis of randomized controlled trials (RCTs).

Setting: Electronic databases, namely EMBASE, PubMed, the Wanfang Data Chinese Database, and the China Knowledge Resource Integrated Database were searched from inception to February 24, 2015

Methods: This review was conducted according to the guidelines of the PRISMA. Only RCTS evaluating the effects of intravenous or oral magnesium on migraine compared with a control group were included.

Results: A total of 21 studies were included. Of which, 11 studies investigated the effects of intravenous magnesium on acute migraine (948 participants) and 10 examined the effects of oral magnesium on migraine prophylaxis (789 participants). Intravenous magnesium significantly relieved acute migraine within $15-45$ minutes, 120 minutes, and 24 hours after the initial infusion (Odd ratios [ORs] $=0.23,0.20$, and 0.25 , respectively). Oral magnesium significantly alleviated the frequency and intensity of migraine (ORs $=0.20$ and 0.27 ).

Limitations: Some of the included studies did not adopt adequate randomization methods.

Conclusions: Intravenous magnesium reduces acute migraine attacks within 15 - 45 minutes, 120 minuts, and 24 hours after the initial infusion and oral magnesium alleviates the frequency and intensity of migraine. Intravenous and oral magnesium should be adapted as parts of multimodal approach to reduce migraine.

Key words: Magnesium, migraine, meta-analysis

Pain Physician 2016; 19:E97-E112

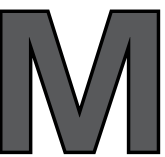

igraine is a common public health concern in contemporary society, with a prevalence rate of $11.7 \%$ (1). Migraine is one of the leading causes of disability (2) and has been associated with increased health care expense $(3,4)$ as well as impaired health-related quality of life (5). Therefore, finding effective approaches for migraine relief is a high priority in clinical settings.

Magnesium deficiency has been strongly associated with migraine attacks $(6,7)$. Several potential mechanisms have been proposed, such as triggered cortical spreading depression (8), decreased release of sub- 
stance $P(9)$, stimulated cerebral artery spasm (10), and an imbalance between mitochondrial energy production and demand (11). Therefore, the clinical effects of magnesium have drawn considerable attention. Previous studies have produced conflicting findings regarding the association of intravenous magnesium and oral magnesium supplements with migraine. Some studies have supported the beneficial effects of the magnesium therapy on acute migraine attacks and migraine prophylaxis (12-14), whereas others have denied any positive relationship between the magnesium therapy and migraine (15-17). A recent meta-analysis of 5 randomized controlled trials (RCTs) (18) demonstrated that intravenous magnesium produced no substantial effect on acute migraine attacks (30 minutes after treatment). However, this review included only a few studies published in English, which could limit its external validity. Moreover, thus far, no meta-analysis has been conducted to evaluate the overall effects of oral magnesium supplements on the prophylaxis of migraine.

We conducted a meta-analysis to confirm the overall effects of intravenous magnesium on acute migraine attacks and oral magnesium supplements on the prophylaxis of migraine by using data of available RCTs published in both English and Chinese.

\section{Methods}

\section{Trial Identification and Data Extraction}

This meta-analysis was conducted according to the preferred reporting items for systematic reviews and meta-analyses statement (19). To identify the articles investigating the effects of intravenous magnesium on acute migraine attacks, we systematically searched the electronic databases, namely EMBASE, PubMed, the Wanfang Data Chinese Database, and the China Knowledge Resource Integrated Database from inception to February 24, 2015. The following combination of search string was used: "migraine" AND "intravenous magnesium" AND "randomized controlled trials." Eligible RCTs reporting the effects of oral magnesium on migraine prophylaxis were identified by searching EMBASE, PubMed, the Wanfang Data Chinese Database, and the China Knowledge Resource Integrated Database from inception to February 24, 2015. The keywords included "migraine" AND "oral magnesium" AND "randomized controlled trials."

Studies were included in the meta-analysis according to the following inclusion criteria: (1) studies having participants $\geq 17$ years diagnosed with migraine,
(2) studies with intravenous magnesium or oral magnesium supplements used as interventions, (3) studies with a control group either inactive or active, (4) studies that have reported the outcomes of migraine, (5) prospective RCTs, and (6) studies that have been published or accepted for publication in English or Chinese by a peer-reviewed journal. Studies involving participants with a diagnosis with menstrual migraine or other types of headache were excluded.

Two raters (HYC and PYC) independently screened the titles and abstracts of potentially eligible articles by using the search strategies described previously. Two authors (HYC and PYC) developed 2 data extraction sheets for studies investigating intravenous magnesium and oral magnesium in migraine, and extracted the data on various factors (Table 1 and 2).

\section{Methodological Quality Assessment}

To confirm the internal validity of each included study, 2 authors (HYC and PYC) individually evaluated potential sources of bias in the studies investigating the effects of intravenous magnesium and oral magnesium on reducing migraine by using the criteria recommended in the Cochrane Handbook for Systematic Review of Intervention 5.1.0 (20).

\section{Statistical Analyses}

Odds ratios (ORs) with $95 \%$ confidence intervals (Cls) were calculated based on the numbers of event and non-event to express the comparison of migraine reduction. For continuous outcomes (i.e., the scores of visual analogues scales and numeric rating scales), we calculated the standardized mean differences and their variances and then converted these values to their corresponding ORs and variances (21). An OR value of less than one denote a negative association between the uses of magnesium and migraine reductions. The probability value of between-study heterogeneity was examined by calculating the Cochran's Q value (22), with $Q$ statistics $<0.05$ representing substantial heterogeneity. The $1^{2}$ value estimates the degree of inconsistency in the study results (22). Roughly, an $I^{2}$ value of $50 \%$ or more reflects substantial heterogeneity, whereas $\mathrm{I}^{2}$ values less than $50 \%$ represent no heterogeneity. To explore the possible reasons for observed heterogeneity, moderator analyses and meta-regression were performed (23). To ensure that sufficient data could be obtained for moderator analyses, the analyses were limited to instances in which groups were represented by at least 2 studies. If the presence of outlying stud- 
Effects of Intravenous and Oral Magnesium on Reducing Migraine

\begin{tabular}{|c|c|c|c|c|c|c|c|c|c|}
\hline & 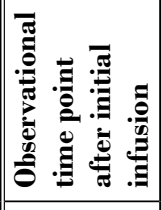 & 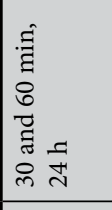 & 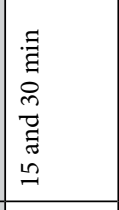 & 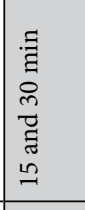 & $\begin{array}{l}\text { 青 } \\
\text { \& } \\
\end{array}$ & \begin{tabular}{|l} 
音 \\
号 \\
\end{tabular} & 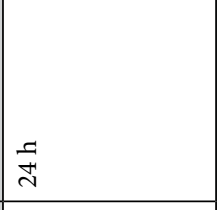 & 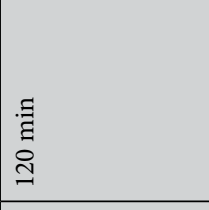 & 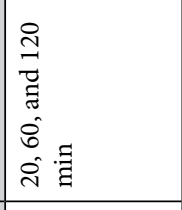 \\
\hline & 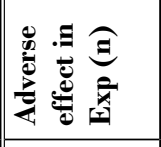 & 5 & 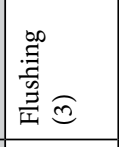 & 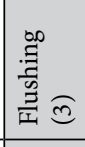 & 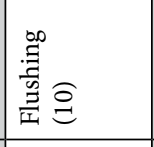 & 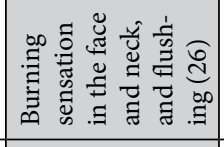 & $\check{z}$ & है & $\begin{array}{l}\widetilde{\Xi} \\
\mathscr{W} \\
\tilde{z} \\
\tilde{Z}\end{array}$ \\
\hline & 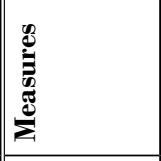 & 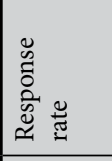 & 恣 & $\stackrel{n}{5}$ & 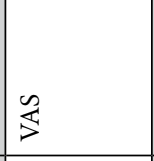 & 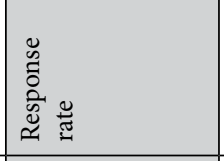 & 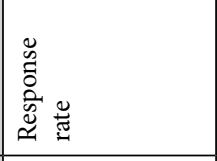 & 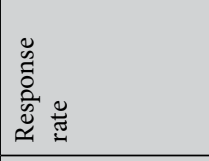 & 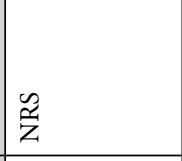 \\
\hline & $\bar{\theta}$ & 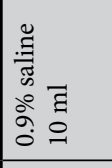 & 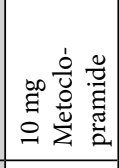 & 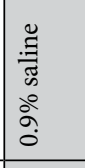 & 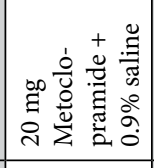 & 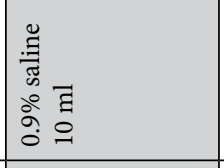 & 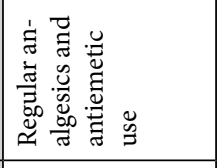 & 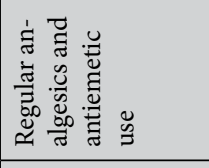 & 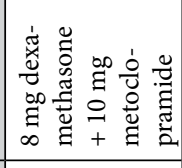 \\
\hline & 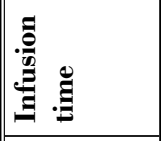 & \begin{tabular}{|l} 
हี \\
है \\
ते \\
\end{tabular} & 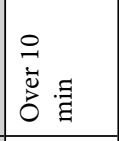 & 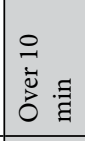 & \begin{tabular}{|l} 
寻 \\
ค月 \\
\end{tabular} & 织 & 音 & 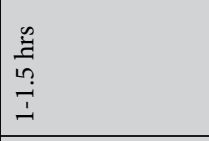 & 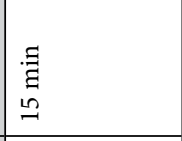 \\
\hline & 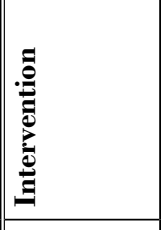 & 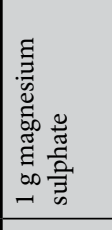 & 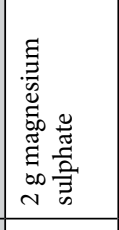 & 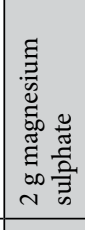 & 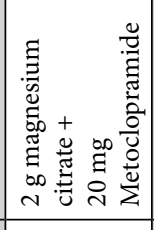 & 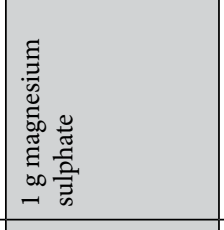 & 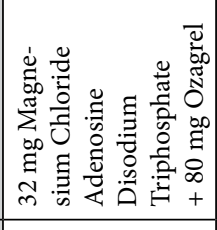 & 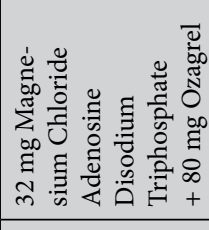 & 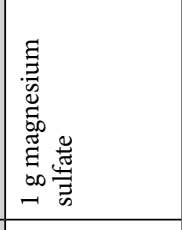 \\
\hline & 。 & $\begin{array}{l}0 \\
i \\
\\
\end{array}$ & \begin{tabular}{|c|}
\multirow{1}{*}{} \\
$\infty$ \\
$\infty$
\end{tabular} & \begin{tabular}{|l}
0 \\
$\infty$ \\
\end{tabular} & $\begin{array}{l}n \\
2 \\
\alpha \\
\alpha\end{array}$ & $\begin{array}{l}0 \\
\infty \\
\end{array}$ & 弚 & बें & $\hat{i}$ \\
\hline & $\frac{8}{4}$ & $\begin{array}{l}4 \\
\infty \\
i \\
i\end{array}$ & 过 & 原 & $\begin{array}{l}0 \\
\infty \\
\infty\end{array}$ & \begin{tabular}{|c}
0 \\
in \\
m
\end{tabular} & in & $\begin{array}{c}n \\
\infty \\
\infty \\
\infty\end{array}$ & $\begin{array}{l}\stackrel{0}{.} \\
\text { m }\end{array}$ \\
\hline & 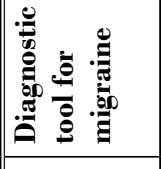 & 突 & 葟 & 式 & 式 & 氙 & 占 & 寻 & 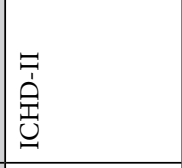 \\
\hline & 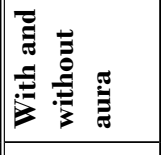 & $\cong$ & $\cong$ & 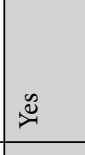 & 光 & 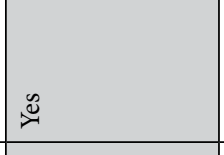 & $\approx$ & $\cong$ & 弚 \\
\hline & 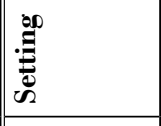 & 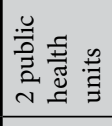 & 외 & 囯 & 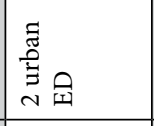 & 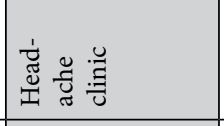 & 光 & క̆ & 贫 \\
\hline & 童 & $\frac{8}{8}$ & 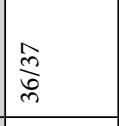 & \begin{tabular}{|l}
0 \\
$\frac{9}{1}$ \\
$i$ \\
$m$ \\
\end{tabular} & 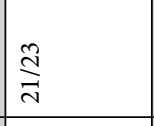 & $\frac{12}{12}$ & $\begin{array}{l}8 \\
8 \\
0 \\
\end{array}$ & $\frac{O}{P}$ & \begin{tabular}{|l}
$m$ \\
$m$ \\
$m$ \\
$m$ \\
$m$
\end{tabular} \\
\hline & 总 & 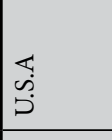 & 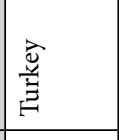 & 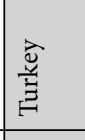 & 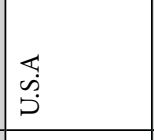 & 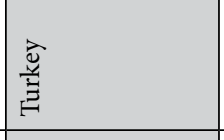 & 莺 & 起 & 馬 \\
\hline & 焉 & 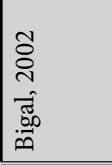 & 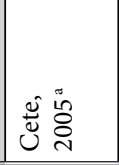 & Uీ & 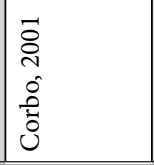 & 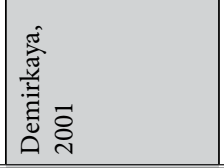 & $\begin{array}{l}m \\
\stackrel{3}{a} \\
3 \\
\exists\end{array}$ & 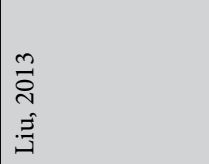 & 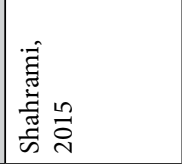 \\
\hline
\end{tabular}


Pain Physician: January 2016; 19:E97-E112

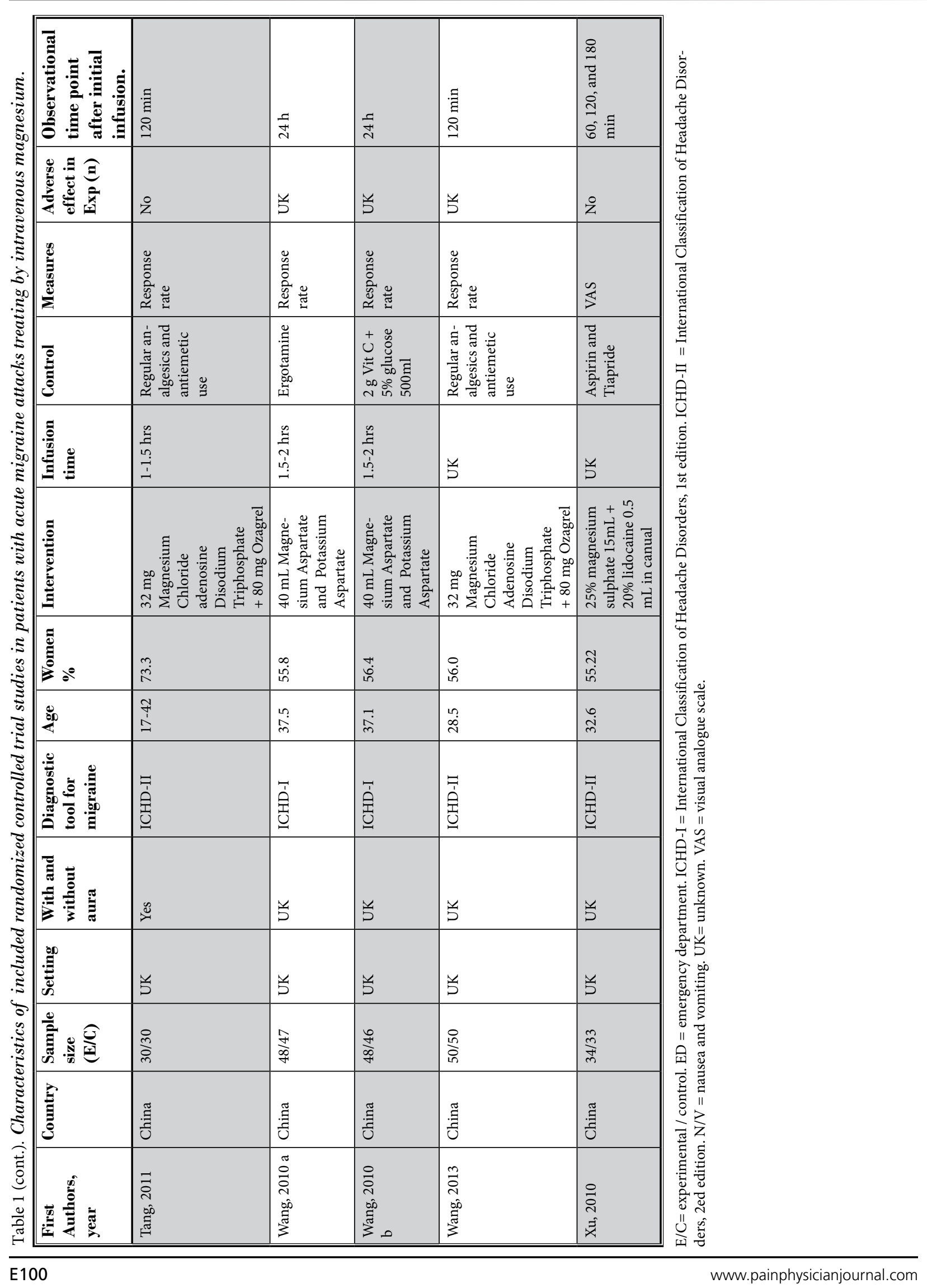




\begin{tabular}{|c|c|c|c|c|c|c|c|c|c|c|c|c|}
\hline 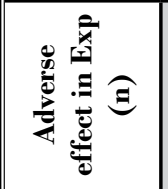 & 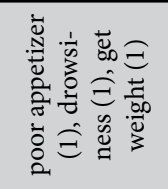 & 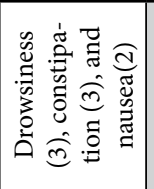 & 岇 & 岇 & 光 & 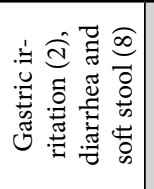 & $\stackrel{\circ}{z}$ & 光 & 光 & 岇 & & 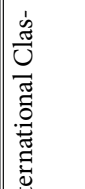 \\
\hline$\overline{8}$ & 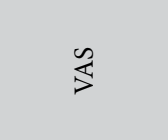 & 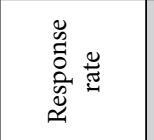 & $\stackrel{5}{>}$ & 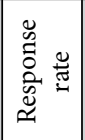 & $\hat{\overrightarrow{0}}$ & $\sum_{>}^{\infty}$ & 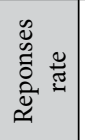 & एक & $\sum_{>}^{5}$ & 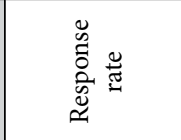 & & \\
\hline $\begin{array}{l}\mathscr{e} \\
\stackrel{\Xi}{0} \\
\stackrel{\Xi}{0}\end{array}$ & 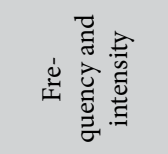 & 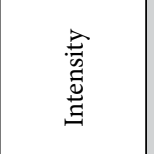 & 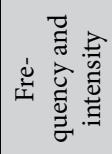 & 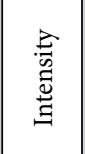 & 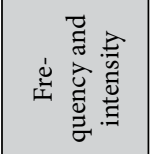 & 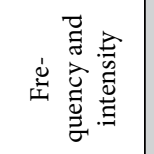 & 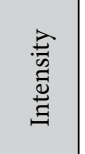 & 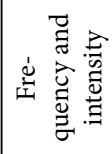 & 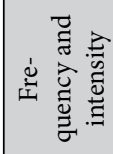 & 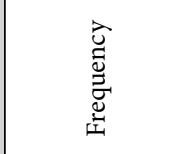 & 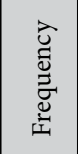 & 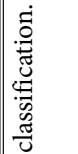 \\
\hline 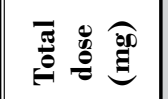 & $\begin{array}{l}\infty \\
\infty \\
\infty \\
\infty\end{array}$ & 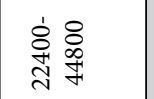 & 离 & 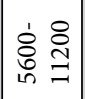 & 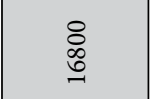 & 京 & $\underset{\sim}{\stackrel{\sim}{~}}$ & ষ্ণ & ষ্ণ & 弚 & : & \\
\hline 总 & $\simeq$ & $\infty$ & $\simeq$ & $r$ & $\infty$ & $\simeq$ & $H$ & $\simeq$ & $\simeq$ & $r$ & $\simeq$ & \\
\hline 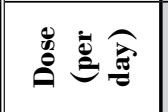 & 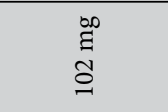 & 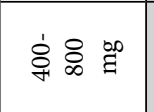 & 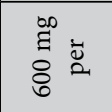 & 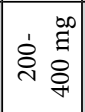 & $\begin{array}{l}\text { bo } \\
\text { a } \\
8 \\
0 \\
0\end{array}$ & $\begin{array}{l}0 \\
0 \\
0 \\
0 \\
0\end{array}$ & 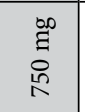 & 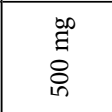 & $\begin{array}{l}\text { bo } \\
\text { हूँ } \\
\text { in }\end{array}$ & za & $\begin{array}{l}\infty \\
\vdots \\
\vdots \\
\vdots \\
\vdots\end{array}$ & \\
\hline $\bar{\theta}$ & 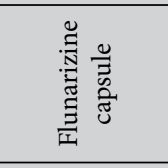 & 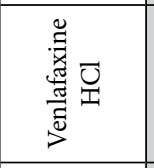 & $\begin{array}{l}\stackrel{8}{\circ} \\
\frac{\tilde{\Xi}}{2} \\
\frac{\pi}{2}\end{array}$ & 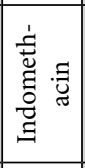 & 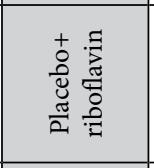 & 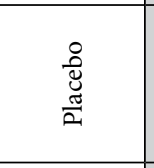 & 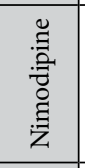 & 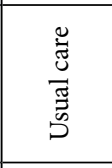 & 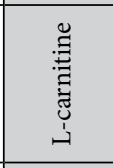 & 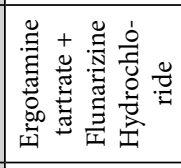 & 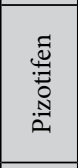 & נֶ, \\
\hline 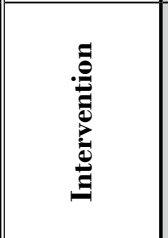 & 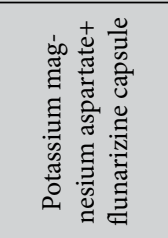 & 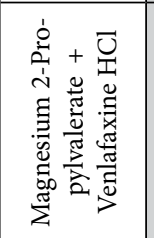 & 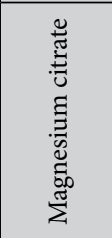 & 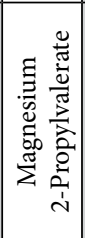 & 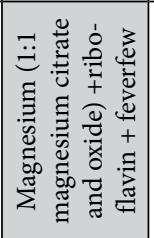 & 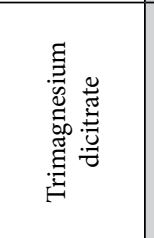 & 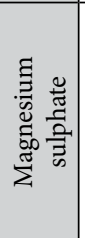 & 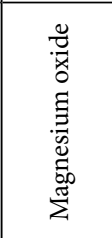 & 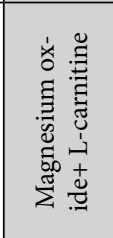 & 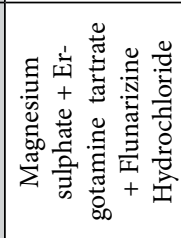 & 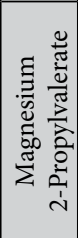 & 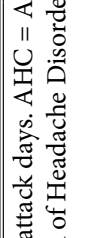 \\
\hline 常 & శ్రి & 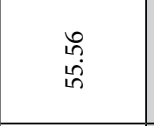 & 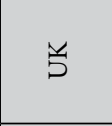 & है & है & $\begin{array}{l}\ddot{H} \\
\stackrel{\infty}{\infty}\end{array}$ & તું & के & 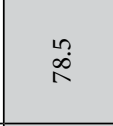 & $\stackrel{\bullet}{\stackrel{i}{i}}$ & $\tilde{n}$ & \\
\hline$\frac{8}{4}$ & ָુ & $\underset{m}{m}$ & $\overrightarrow{+1}$ & है & 光 & 字 & $\stackrel{n}{m}$ & ঙं & $\stackrel{\sim}{m}$ & $\stackrel{\infty}{d}$ & $\begin{array}{l}\infty \\
\stackrel{m}{m} \\
\end{array}$ & \\
\hline 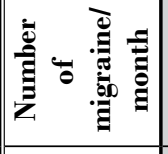 & $\stackrel{\wedge}{\wedge}$ & 岇 & 岇 & 岇 & $\stackrel{\infty}{\sim}$ & 弚 & $\breve{s}$ & $\lambda$ & $\lambda$ & 岇 & $\lambda$ & 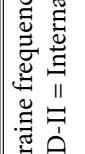 \\
\hline 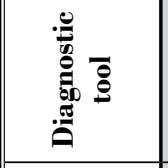 & 寻 & 完 & 寻 & 岁 & 空 & 灾 & כ̆ & 灾 & 灾 & 灾 & 灾 & \\
\hline 曾 & $\frac{f}{b}$ & $\begin{array}{l}\text { 点 } \\
\stackrel{H}{H}\end{array}$ & 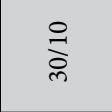 & $\stackrel{\stackrel{ }{\sharp}}{\frac{7}{4}}$ & 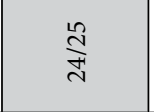 & $\stackrel{\substack{m \\
q}}{q}$ & $\underset{\substack{f \\
\infty}}{\infty}$ & 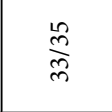 & 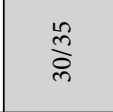 & 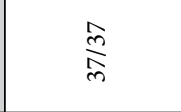 & $\stackrel{\text { in }}{=}$ & \\
\hline ق & శี & శี & $\begin{array}{l}\text { 氙 } \\
\text { 音 }\end{array}$ & ]ี & 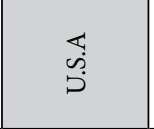 & 苛 & 节 & 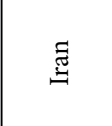 & 急 & ]ี & శ్ & 莺 \\
\hline 焉 & : & 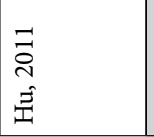 & 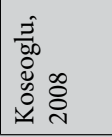 & 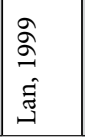 & 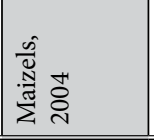 & 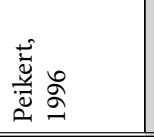 & 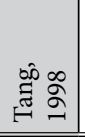 & 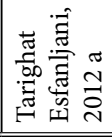 & 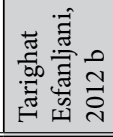 & 论 & 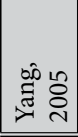 & Ir \\
\hline
\end{tabular}


ies with results that conflict with the rest of the studies was observed, a sensitivity analysis was performed (20). In addition, sensitivity analyses were carried out to further examine whether the use of diagnostic criteria for migraine before enrollment influenced the effects of magnesium on reducing migraine. In case multiple treatments or controls were used in one study, we divided the shared intervention or control groups into 2 groups and then compared to their counterpart. For dichotomous outcomes, the number of events and the total number of patients were divided. For continuous outcomes, only the total number of patients were divided, and the means and standard deviations were left unchanged (20). Because of a higher degree of random variation, studies with smaller samples yielded a wider distribution than studies with larger samples did, thus causing asymmetry in a funnel plot $(24,25)$. Because this meta-analysis included a limited number of studies, publication bias was examined using the Egger's intercept test (24). All analyses were performed by an inverse variance random-effect model (26) using Comprehensive Meta-Analysis software, version 2.0 (Biostat, Englewood, New Jersey).

\section{Results}

\section{Search Results}

With regard to the effects of intravenous magnesium on acute migraine attacks, the literature search initially identified 51 articles. Among these, 10 duplicate articles were excluded using Thomson Reuters Endnote software X7. Thirty-one studies were excluded after initial review, because the participants and interventions in those studies were irrelevant to the topic of the present study (e.g., people with menstrual migraine, unrelated to the treatment of intravenous magnesium), and those articles were either not based on RCTs or not published in English or Chinese (e.g., Portuguese). Fourteen articles were maintained for further screening. Three studies were excluded because one study (27) provided insufficient data for computing an effect size even after contacting the authors, and 2 studies $(16,28)$ enrolled participants with migraine and other types of headache. Finally, 11 studies $(12,13,15,29-36)$ were included for the meta-analysis. Two studies $(29,35)$ employed a 3-arm study design resulting in 13 trials for final analyses (Fig. 1).

Regarding to the effects of intravenous magnesium on acute migraine attacks, the literature search initially identified 51 articles. Among these, 9 duplicate articles were excluded using Thomson Reuters Endnote software X7. Thirty-six studies were excluded after initial review, because the participants and interventions in those studies were irrelevant to the topic of the present study (e.g., unrelated to the treatment of oral magnesium, and inclusion of children and adolescents), and those articles were not based on RCTs. Fourteen articles were maintained for further screening. Six studies were excluded because one study (37) used oral magnesium in both experimental and control groups, and 5 studies (38-42) did not evaluate the outcomes immediately following the treatments. Ten studies $(14,17,43-50)$ evaluated the effects of oral magnesium supplements on prophylaxis of migraine. One study (51) employed a 4-arm study design resulting in 11 trials for analyses (Fig. 2).

\section{Study Characteristics}

Table 1 presents summaries of the study characteristics of the effects of intravenous magnesium supplements on migraine. Among the included 13 trials, $(12,13,15,29-36)$ study sample sizes ranged from 15 to 60 with a total of 948 randomized patients. Seven trials were conducted in China. To diagnose migraine, 8 trials employed the International Classification of Headache Disorders, 1st edition (ICHD-I) and 5 trials used ICHD, 2nd edition (ICHD-II). Six trials used intravenous magnesium combined with other therapies as the treatment arm. Two types of control conditions were used for comparison: inactive groups ( $0.9 \%$ saline) and active groups (e.g., metoclopramide, prochlorperazine, aspirin, tiapride, and ergotamine). Eight trials reported adverse effects such as flushing and burning sensation in the face, neck, and the intravenous site. Eight trials used the response rate for measuring the change of pain.

Table 2 shows summaries of the study characteristics of the effects of oral magnesium supplements on migraine. Eleven trials $(14,17,43-50)$ involving 789 participants were included. Six trials were conducted in China. Five trials used oral magnesium combined with other therapies (e.g., ergotamine) as the treatment arm. Two types of control conditions were used for comparison: inactive groups (placebo) and active groups (e.g., venlafaxine $\mathrm{HCl}$, flunarizine hydrochloride, riboflavin, pizotifen, and ergotamine). Seven trials employed the ICHD-I, 2 used the ICHD-II, and one used the Ad Hoc Committee on classification of headache classification to confirm the diagnosis of migraine. One trial did not report whether participants met the diagnostic criteria for migraine (50). Of the 11 included trials, magnesium 2-propylvalerate was used in 3 trials, 2 used magnesium 


\section{Search of electronic databases}

11 EMBASE

4 PubMed

16 Wanfang Data Chinese database

19 China Knowledge Resource

Integrated Database

4 of additional records identified through other sources

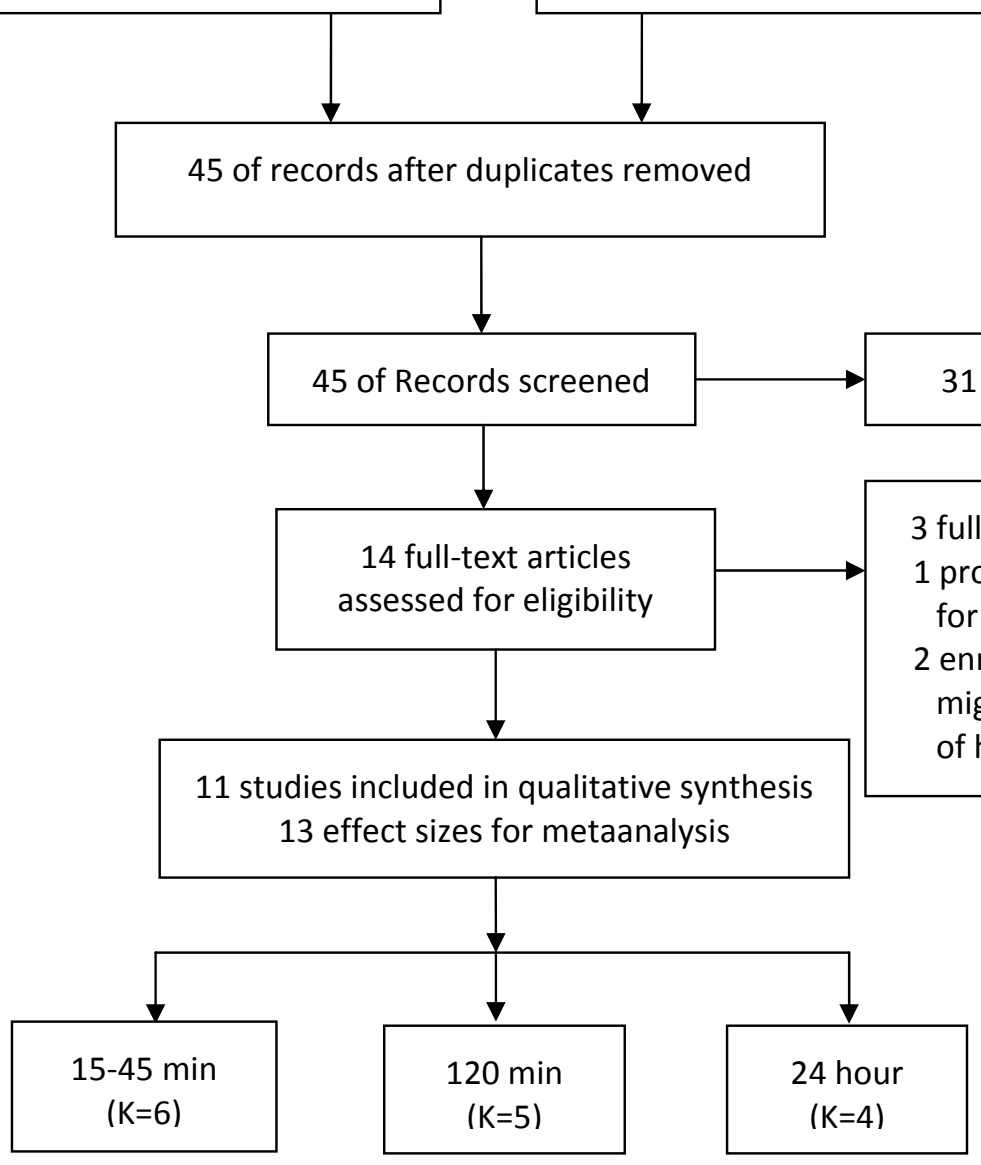

Fig. 1. Preferred reporting items for systematic reviews and meta-analyses 2009 flow diagram (intravenous magnesium).

oxide, and 2 used magnesium sulphate. Other formulations including magnesium citrate, the combination of magnesium oxide and citrate, potassium magnesium, and trimagnesium dicitrate were respectively used in 4 trials. The mean treatment duration was 9 weeks, ranging from 4 weeks to 12 weeks. Five trials reported adverse effects including gastrointestinal symptoms, dizziness, and drowsiness.

\section{Assessment of Study Bias}

The methodological quality of the included studies is reported in Table 3. Regarding studies on intravenous magnesium, all trials achieved the selective reporting. Approximately $40 \%$ of the studies $(k=5)$ generated a random sequence with correct approaches and blinded participants and personnel. Two studies blinded outcome assessors. Only one study concealed allocation or 


\section{Search of electronic databases \\ 17 EMBASE \\ 5 PubMed \\ 16 Wanfang Data Chinese database \\ 19 China Knowledge Resource \\ Integrated Database}

3 of additional records identified through other sources

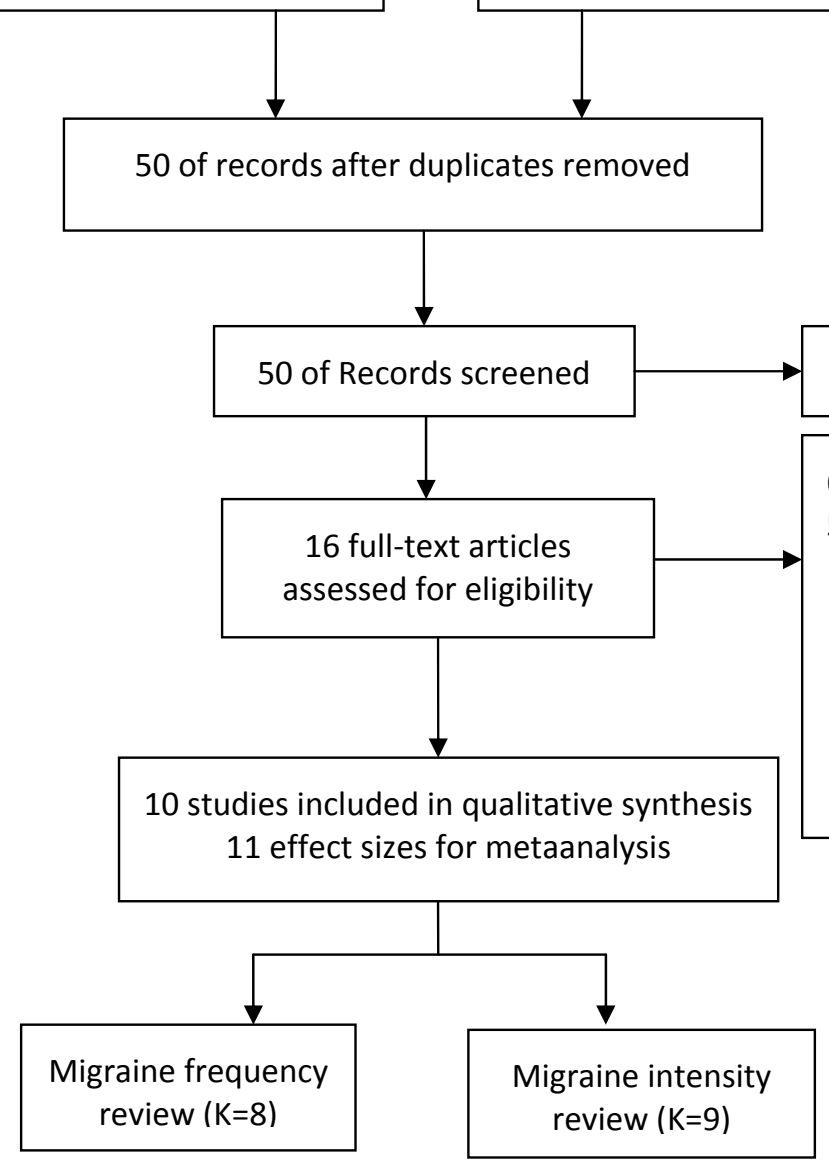

34 of records excluded

6 full-text articles excluded

5 did not evaluate the outcomes immediately after the completion of treatments

1 used oral magnesium in both experimental and control group

Fig. 2. Preferred reporting items for systematic reviews and meta-analyses 2009 flow diagram (oral magnesium).

addressed incomplete outcome data. Regarding studies on oral magnesium supplements, all trials achieved the selective reporting. Nearly $20 \%$ of the studies ( $k=$ 2) generated a random sequence through appropriate approaches, blinded participants and personnel, and addressed incomplete outcome data. Only one study blinded outcome assessors. None of studies concealed allocation.

\section{Overall Effects of Intravenous Magnesium on Acute Migraine Attacks}

Among the 13 included trials, 6, 5, and 4 of them investigated the effects of intravenous magnesium on acute migraine attacks within 15 - 45 minutes, 120 minutes, and 24 hours following the initial infusion, respectively.

Regarding the effects observed within 15 - 45 min- 
utes, the effect sizes are illustrated in Fig. 3A. The data favored intravenous magnesium for acute migraine attacks with a pooled OR of $0.23(95 \% \mathrm{Cl}=0.09$ to 0.58 , $P=0.002)$. Calculation of the $I^{2}$ value $(73.2 \%)$ and Cochran's $Q$ value (18.7) resulted in the identification of heterogeneity $(P=0.002)$.

With regard to the effects observed within 120 minutes, the pooled OR of $0.20(95 \% \mathrm{Cl}=0.10$ to 0.40 , $P<0.001$ ) was found (Fig. 3B). The $\mathrm{I}^{2}$ and Cochran's Q values indicated homogeneity across all the included studies $\left(\mathrm{Q}=7.12 ; P=0.13, \mathrm{I}^{2}=43.8 \%\right)$.

Fig. $3 C$ shows the results of the treatment effects observed within 24 hours following the initial infusion $(30,35)$. The pooled OR of $0.25(95 \% \mathrm{Cl}=0.10$ to $0.60, P=0.002$ ) was observed. We found evidence of between-study heterogeneity $\left(\mathrm{Q}=8.37, P=0.04, \mathrm{I}^{2}=\right.$ $64.2 \%)$.

\section{Overall Effects of Oral Magnesium Supplements on Migraine Frequency and Intensity}

Eight trials $(14,17,44-48)$ that investigated the effects of oral magnesium supplements on the frequency of migraine were included in the analysis (Fig. 4A). Oral magnesium caused a significant reduction in the frequency of migraine (pooled $\mathrm{OR}=0.20,95 \% \mathrm{Cl}=0.05$ to $0.89, P=0.04)$. There was evidence for heterogeneity across all the included studies $\left(\mathrm{Q}=98.22, P<0.001, \mathrm{I}^{2}\right.$ $=92.87$ ).

Nine trials $(14,17,43-45,48)$ investigated the effect

Table 3. Risk of methodological bias score of the studies.

\begin{tabular}{|c|c|c|c|c|c|c|}
\hline First Authors & $\begin{array}{c}\text { Random } \\
\text { sequence } \\
\text { generation }\end{array}$ & $\begin{array}{c}\text { Allocation } \\
\text { concealment }\end{array}$ & $\begin{array}{c}\text { Blinding of } \\
\text { participants and } \\
\text { personnel }\end{array}$ & $\begin{array}{c}\text { Blinding } \\
\text { of outcome } \\
\text { assessment }\end{array}$ & $\begin{array}{c}\text { Incomplete } \\
\text { outcome data } \\
\text { addressed }\end{array}$ & $\begin{array}{l}\text { Selective } \\
\text { reporting }\end{array}$ \\
\hline \multicolumn{7}{|l|}{ Intravenous magnesium } \\
\hline Bigal, 2002 & + & $?$ & + & $?$ & $?$ & + \\
\hline Cete, 2005 a & + & $?$ & + & $?$ & $?$ & + \\
\hline Cete, 2005 b & + & $?$ & + & $?$ & $?$ & + \\
\hline Corbo, 2001 & + & + & + & + & $?$ & + \\
\hline Demirkaya, 2001 & $?$ & $?$ & $?$ & $?$ & $?$ & + \\
\hline $\mathrm{Li}, 2013$ & - & $?$ & $?$ & $?$ & $?$ & + \\
\hline Liu, 2013 & $?$ & $?$ & $?$ & $?$ & $?$ & + \\
\hline Shahrami, 2015 & + & $?$ & + & + & + & + \\
\hline Tang, 2011 & $?$ & $?$ & $?$ & $?$ & $?$ & + \\
\hline Wang, $2010 \mathrm{a}$ & $?$ & $?$ & $?$ & $?$ & $?$ & + \\
\hline Wang, $2010 \mathrm{~b}$ & $?$ & $?$ & $?$ & $?$ & $?$ & + \\
\hline Wang, 2013 & $?$ & $?$ & $?$ & $?$ & $?$ & + \\
\hline $\mathrm{Xu}, 2010$ & $?$ & $?$ & $?$ & $?$ & $?$ & + \\
\hline \multicolumn{7}{|l|}{ Oral magnesium } \\
\hline Bian, 2013 & $?$ & $?$ & $?$ & $?$ & $?$ & + \\
\hline $\mathrm{Hu}, 2011$ & $?$ & $?$ & $?$ & $?$ & $?$ & + \\
\hline Koseoglu, 2008 & + & $?$ & $?$ & $?$ & $?$ & + \\
\hline Lan, 1999 & $?$ & $?$ & $?$ & $?$ & $?$ & + \\
\hline Tang, 1998 & $?$ & $?$ & $?$ & $?$ & $?$ & + \\
\hline Tarighat Esfanjani, 2012 a & $?$ & $?$ & $?$ & $?$ & + & + \\
\hline Tarighat Esfanjani, $2012 \mathrm{~b}$ & $?$ & $?$ & $?$ & $?$ & + & + \\
\hline Maizels, 2004 & $?$ & $?$ & + & + & - & + \\
\hline Peikert, 1996 & $?$ & $?$ & $?$ & $?$ & $?$ & + \\
\hline Wang, 2001 & $?$ & $?$ & $?$ & $?$ & $?$ & + \\
\hline Yang, 2005 & + & $?$ & + & $?$ & - & + \\
\hline
\end{tabular}

$+=$ low risk; - = high risk; ? = unclear risk of bias. 
Study name

\begin{tabular}{|c|c|c|c|c|c|}
\hline & $\begin{array}{l}\text { Odds } \\
\text { ratio }\end{array}$ & $\begin{array}{l}\text { Lower } \\
\text { limit }\end{array}$ & $\begin{array}{l}\text { Upper } \\
\text { limit }\end{array}$ & \multicolumn{2}{|c|}{ Z-Value p-Value } \\
\hline 2 & 57 & 0.105 & 1.210 & -1.653 & 0.098 \\
\hline Cete, 2005 a & 313 & 110 & 0.889 & -2.181 & .029 \\
\hline Cete, 2005 b & 339 & 0.121 & 0.947 & -2.064 & 0.039 \\
\hline Corbo, 2001 & 0.957 & 0.327 & 2.799 & -0.080 & 0.936 \\
\hline Demirkaya, 2001 & 011 & .001 & 0.136 & -3.513 & 0.000 \\
\hline Shahram, 2015 & 0.077 & 0.030 & 0.199 & -5.292 & 0.000 \\
\hline & 0.227 & 0.089 & 0.577 & -3.118 & 0.00 \\
\hline
\end{tabular}

\section{Odds ratio and $95 \% \mathrm{Cl}$}

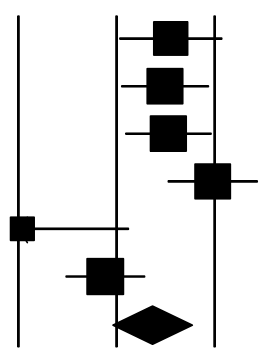

$\begin{array}{lllll}0.01 & 0.1 & 1 & 10 & 100\end{array}$

Favours IVmagnesium Favours Control
Study name

\begin{tabular}{|c|c|c|c|c|c|}
\hline & $\begin{array}{l}\text { Odds } \\
\text { ratio }\end{array}$ & $\begin{array}{c}\text { Lower } \\
\text { limit }\end{array}$ & $\begin{array}{l}\text { Upper } \\
\text { limit }\end{array}$ & Z-Value & p-Value \\
\hline Liu, 2013 & 0.354 & 0.085 & 1.476 & -1.426 & 0.15 \\
\hline Shahrami, 2015 & 0.219 & 0.090 & 0.531 & -3.361 & 0.00 \\
\hline Tang, 2011 & 0.357 & 0.064 & 2.007 & -1.169 & 0.2 \\
\hline Wang, 2013 & 0.315 & 0.093 & 1.070 & -1.852 & 0.0 \\
\hline \multirow[t]{2}{*}{ Xu, 2010} & 0.060 & 0.022 & 0.163 & -5.555 & 0.0 \\
\hline & 0.197 & 0.097 & 0.401 & -4.473 & 0.0 \\
\hline
\end{tabular}

Odds ratio and $95 \% \mathrm{Cl}$

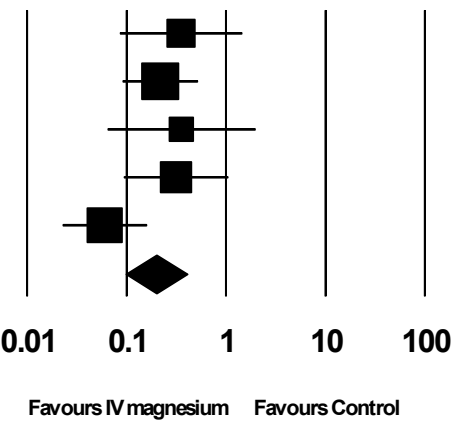

Study name

Statistics for each study

Odds ratio and $95 \% \mathrm{Cl}$

Odds Lower Upper

ratio limit limit Z-Value $p$-Value

$\begin{array}{llllll}\text { Bigal, 2002 } & 0.500 & 0.229 & 1.091 & -1.742 & 0.082 \\ \text { Li, 2013 } & 0.357 & 0.105 & 1.210 & -1.653 & 0.098 \\ \text { Wang, 2010 a } & 0.269 & 0.091 & 0.799 & -2.363 & 0.018 \\ \text { Wang, 2010 b } & 0.060 & 0.018 & 0.204 & -4.505 & 0.000 \\ & 0.247 & 0.102 & 0.598 & -3.096 & 0.002\end{array}$

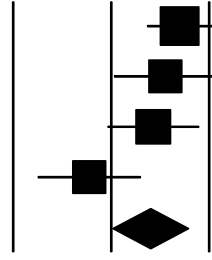

$\begin{array}{lllll}0.01 & 0.1 & 1 & 10 & 100\end{array}$

Favours IV magnesium Favours Control

Fig. 3. Forest plots for studies measuring the effect of intravenous magnesium on acute migraine attacks in the time frames of 15-45 $\min (A), 120 \mathrm{~min}(B)$, and (C) $24 \mathrm{~h}$ (D) following the initial infusion. 


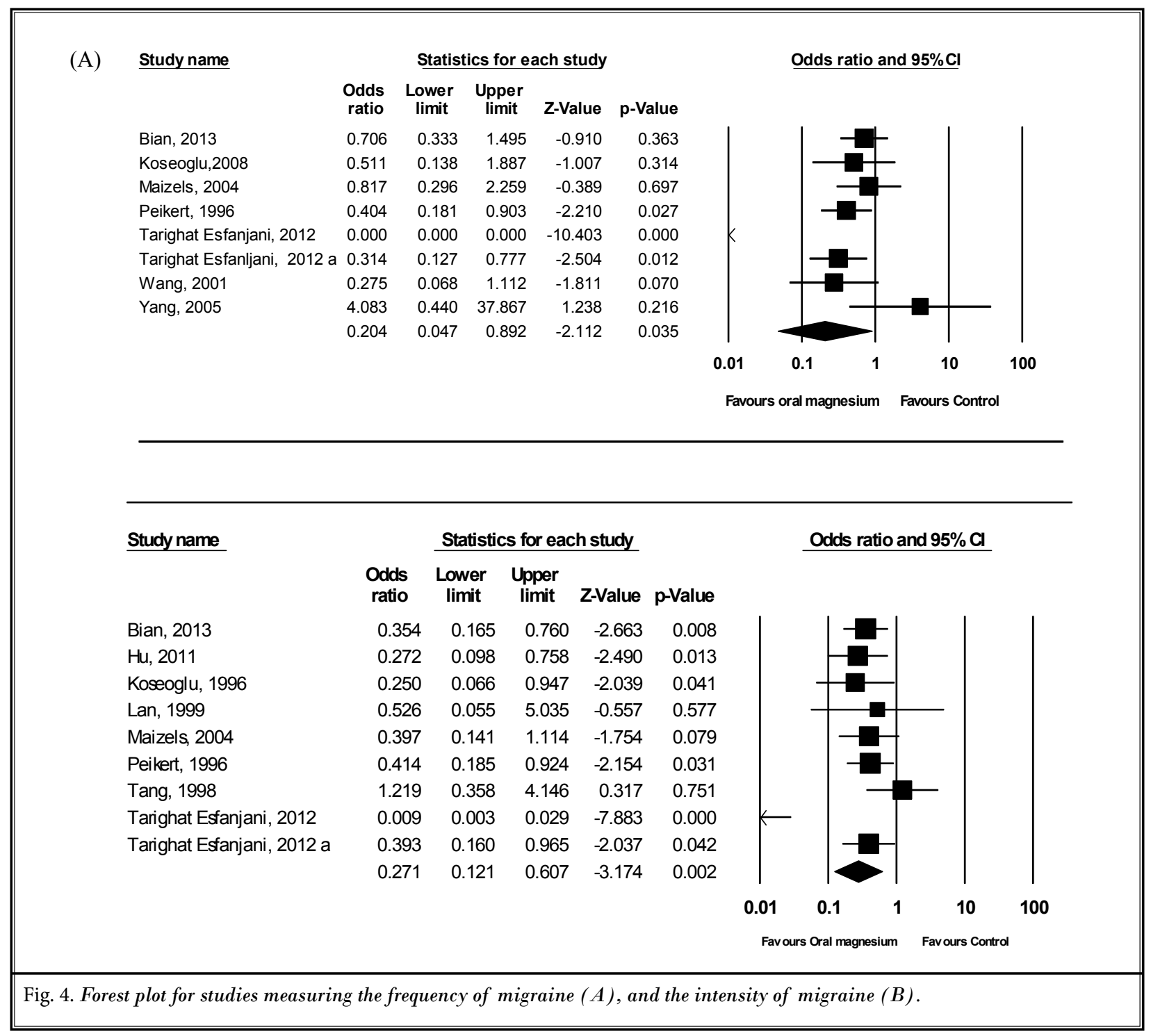

of oral magnesium supplements on the intensity of migraine (Fig. 4B). The pooled OR was $0.27(95 \% \mathrm{Cl}=0.12$ to 0.61 ), with the $\mathrm{I}^{2}$ and Cochran's $\mathrm{Q}$ value indicating heterogeneity across all the included studies $(Q=41.17$, $\left.P<0.001, I^{2}=80.57\right)$.

\section{Moderator Analyses and Meta-regression}

As seen in Table 4, in determining the moderator effects of intravenous magnesium within $15-45$ minutes after the initial infusion, the percent of women was associated with a significantly increased migraine attack, with an OR of $0.05(95 \% \mathrm{Cl}=0.02$ to $0.08, P=$ 0.003 ) per one point. Although intravenous magnesium combined with other treatments yielded fewer reduc- tions in acute migraine attack than magnesium alone (OR $=0.61$ and 0.33 ), the difference was not statistically significant $(P=0.52)$. In addition, the effects of intravenous magnesium combined with other therapies on reducing migraine was found to be nonsignificant $(95 \%$ $\mathrm{Cl}=0.36$ to 1.02 ).

Regarding the moderating effects of the intravenous magnesium within 24 hours after the initial infusion, Increased age was associated with a significant reduction of migraine ( $O R=-0.14,95 \% \mathrm{Cl}=-0.28$ to $-0.004, P=0.04)$. The percentage of women correlated to a significantly increased migraine attack (OR $=0.18$, $95 \% \mathrm{Cl}=0.02$ to $0.34, P=0.03$ ).

In regard with the moderating effects of oral mag- 
Pain Physician: January 2016; 19:E97-E112

Table 4. Moderator analyses and meta-regressions.

\begin{tabular}{|c|c|c|c|c|}
\hline Parameter & $\overline{\mathbf{k}}$ & Point estimate & $95 \%$ CI & $\boldsymbol{P}$ \\
\hline Intravenous magnesium 15-45 min & 6 & & & \\
\hline Age & 6 & 0.04 & -0.18 to 0.26 & .73 \\
\hline Percentage of women & 6 & 0.05 & 0.02 to 0.08 & .003 \\
\hline \multicolumn{5}{|l|}{ Magnesium combined with other therapies } \\
\hline Yes & 4 & 0.61 & 0.36 to 1.02 & .52 \\
\hline No & 4 & 0.33 & 0.05 to 2.00 & \\
\hline \multicolumn{5}{|l|}{ Types of control group } \\
\hline Active & 2 & 0.15 & 0.04 to 0.60 & .51 \\
\hline Inactive & 4 & 0.28 & 0.08 to 0.97 & \\
\hline \multicolumn{5}{|l|}{ Blinding of outcome assessor } \\
\hline High or unclear risk of bias & 4 & 0.23 & 0.09 to 0.60 & .95 \\
\hline Low risk of bias & 2 & 0.27 & 0.02 to 3.17 & \\
\hline Intravenous magnesium $24 \mathrm{~h}$ & 4 & & & \\
\hline Age & 4 & -0.14 & -0.28 to -0.004 & .04 \\
\hline Percentage of women & 3 & 0.18 & 0.02 to 0.34 & .03 \\
\hline \multicolumn{5}{|l|}{ Types of control group } \\
\hline Active & 2 & 0.31 & 0.14 to 0.69 & .65 \\
\hline Inactive & 2 & 0.18 & 0.02 to 1.46 & \\
\hline Oral Magnesium for migraine frequency & 8 & & & \\
\hline Age & 7 & 0.08 & -0.14 to 0.30 & .48 \\
\hline Percentage of women & 8 & -0.11 & -0.48 to 0.09 & .26 \\
\hline Treatment dosage & 7 & -0.0006 & -0.0002 to 0.0001 & .51 \\
\hline \multicolumn{5}{|l|}{ Magnesium combined with other therapies } \\
\hline Yes & 4 & 0.48 & 0.002 to 3.62 & .34 \\
\hline No & 4 & 0.08 & 0.31 to 0.86 & \\
\hline \multicolumn{5}{|l|}{ Types of control group } \\
\hline Active & 5 & 0.58 & 0.31 to 1.10 & .17 \\
\hline Inactive & 3 & 0.02 & 0.00 to 2.33 & \\
\hline \multicolumn{5}{|l|}{ Region of study } \\
\hline China & 3 & 0.72 & 0.24 to 2.18 & .10 \\
\hline Other countries ${ }^{a}$ & 5 & 0.08 & 0.009 to 0.83 & \\
\hline Oral Magnesium for migraine intensity & 9 & & & \\
\hline Age & 7 & 0.02 & -0.07 to 0.12 & .61 \\
\hline Percentage of women & 8 & -0.04 & -0.14 to 0.05 & .32 \\
\hline Total dosage & 9 & -0.0003 & -0.0008 to 0.0002 & .28 \\
\hline \multicolumn{5}{|l|}{ Magnesium combined with other therapies } \\
\hline Yes & 4 & 0.35 & 0.10 to 1.21 & .57 \\
\hline No & 5 & 0.22 & 0.07 to 0.70 & \\
\hline \multicolumn{5}{|l|}{ Types of control group } \\
\hline Active & 6 & 0.41 & 0.27 to 0.63 & .25 \\
\hline Inactive & 3 & 0.10 & 0.01 to 1.07 & \\
\hline \multicolumn{5}{|l|}{ Region of study } \\
\hline China & 4 & 0.44 & 0.23 to 0.83 & .22 \\
\hline Other countries ${ }^{b}$ & 5 & 0.18 & 0.05 to 0.66 & \\
\hline
\end{tabular}

Abbreviation: $\mathrm{CI}$ = confidence interval. a = Turkey, America, Germany, and Iran. b = Turkey, America, Germany, and Iran. 
nesium, although studies conducted in China yielded fewer reductions in pain frequency and intensity (OR $=0.72$ and 0.44$)$ than did studies conducted in other countries $(O R=0.08$ and 0.18$)$, the differences were not statistically significant $(P=0.10$ and 0.22$)$. Oral magnesium alone had greater reductions in the frequency and intensity of migraine ( $O R=0.08$ and 0.22 ) than magnesium combined with other treatments $(O R=0.48$ and $0.35)$; however, the differences were not statistically significant ( $P=0.34$ and 0.57$)$. Most importantly, the effects of oral magnesium combined with other therapies on reducing the frequency and intensity of migraine were not statistically significant $(95 \% \mathrm{Cl}=0.002$ to 3.62 and 0.10 to 1.21 ). As seen in Table 4 , no moderating effect was found for the effects of oral migraine on the frequency and intensity of migraine.

\section{Sensitivity Analyses}

Sensitivity analyses showed that the adjusted OR of $0.40(95 \% \mathrm{Cl}=0.28$ to 0.57$)$ was observed in the studies examining the effects of oral magnesium on migraine frequency after removing the study with the smallest value of OR. Substantial homogeneity was observed across the included studies $\left(Q=4.37, P=0.74, I^{2}=0 \%\right)$. With regard to the studies investigating oral magnesium on migraine intensity, the adjusted OR was 0.53 $(95 \% \mathrm{Cl}=0.34$ to 0.81$)$ indicating homogeneity across the included studies ( $\left.Q=7.05, P=0.32, I^{2}=14.9 \%\right)$ after omitting the study with the smallest value of $O R$.

After excluding the trial without reporting the diagnostic criteria for migraine, sensitivity analysis revealed that the adjusted $\mathrm{OR}$ was $0.23(95 \% \mathrm{Cl}=0.10$ to 0.52 ) indicating that the effect of oral magnesium on the intensity of migraine was not influenced by the use of diagnostic criteria for migraine before enrollment.

\section{Publication Bias}

For studies that examined the effects of intravenous magnesium on acute migraine attacks within 15 - 45 minutes, 120 minutes, and 24 hours after the initial infusion, no significant publication bias has been observed $(P=0.42,0.36$, and 0.29 , respectively). Regarding the effects of oral magnesium supplements on the frequency and intensity of migraine, no publication bias has been detected ( $P=0.33$ and 0.78 , respectively).

\section{Discussion}

This meta-analysis suggests that intravenous magnesium and oral magnesium supplements produced substantial effects on migraine. Although this meta- analysis could not directly determine how intravenous and oral magnesium improved migraine through the aforementioned mechanisms (6,8-11), the findings of the meta-analysis indirectly support the positive role of magnesium in the acute treatment and prevention of migraine.

Our findings revealed that intravenous magnesium yielded beneficial effects on alleviating acute migraine immediately (15 - 45 minutes) after the initial infusion. In addition, the intermediate (120 minutes) and longterm (24 hours) effects of intravenous magnesium on acute migraine attacks could be observed. Compared with the previous meta-analysis (18), the present metaanalysis examined clinically more relevant effects of magnesium, applied more specific inclusion criteria, and more rigorously assessed the quality of the included studies with 2 independent raters. Thus, the overall results of this meta-analysis should be considered credible.

Because some of the included studies combined intravenous or oral magnesium therapy with other therapies as the interventions, it was difficult to determine whether the beneficial effects on migraine were derived from the magnesium or other therapies. Compellingly, our findings of the moderator analyses showed that both types of studies (those that used intravenous or oral magnesium alone, and those that used the magnesium combined with other therapies [e.g., metoclopramide, ozagrel, and Chinese herbs]) yielded similar treatment effects on alleviating migraine (see Table 4). We also found that the effects of intravenous or oral magnesium combined with other therapies on reducing migraine were not statistically significant. In clinical settings, migraineurs often receive various treatment approaches simultaneously to mitigate their migraine $(52,53)$. Our findings support the beneficial effects of intravenous and oral magnesium on acute migraine attacks and the prophylaxis of migraine, respectively, regardless of whether magnesium is combined with other therapies. However, because of the inclusion of small-scale studies, our findings should be interpreted with caution.

In the meta-regression, the percentage of women was independently associated with treatment effects of intravenous magnesium on alleviating migraine within 15 - 45 minutes, and age and the percentage of women were associated with treatment effects of intravenous magnesium on alleviating migraine within 24 hours. Despite women being more likely to experience migraine than men, and despite the occurrences of migraine ris- 
ing through early adult life and declining in the late 40s and early 50s (54-56), no evidence has directly demonstrated the association among age, gender, and the use of magnesium supplements in patients with migraine. Moreover, we found pooled ORs with relatively wide $95 \% \mathrm{Cls}$ as a result of small-size studies, indicating that the precision of the OR is not optimal. Therefore, our results must be interpreted with caution. Future studies are warranted to investigate this concern.

It is worthwhile to point out that the included studies which investigated the effects of oral magnesium on migraine prophylaxis lacked for standardized treatment protocol as we found that the dosages and the formulations of migraine varied widely. Therefore, not only the optimal dosages but also the effective formulations of magnesium treatment could not be synthesized from our reviews. Future RCTs should focus on exploring the effective dosage and formulation so that the standardization of treatment dosages could be established.

Certain limitations of the present meta-analysis must be acknowledged. Although we conducted a comprehensive literature review, deployments of different search strategies may resulted in selection bias. Some of the included studies did not adopt adequate randomization methods; therefore, our findings should be interpreted with caution. However, there are several strengths in this meta-analysis. First, this meta-analysis included a large sample size. Second, including only RCTs contributed to high internal validity. Third, the inclusion of both Chinese and English RCTs increases the external validity of this review.

\section{Conclusion}

In conclusion, this is the first meta-analysis to evaluate the overall effects of intravenous and oral magnesium on acute migraine attacks and the prophylaxis of migraine, respectively. We confirmed that intravenous magnesium has beneficial effects in relieving acute migraine attacks and that oral magnesium supplements alleviate the frequency and intensity of migraine. Thus, we suggest that intravenous and oral magnesium should be considered as adjunctive therapies for managing acute migraine attacks and the prophylaxis of migraines, respectively. Specifically, additional RCTs in which adequate randomization methods are used for evaluating the effects of intravenous magnesium on acute migraine attacks and oral magnesium on migraine prophylaxis are warranted.

\section{References}

1. Lipton RB, Bigal ME, Diamond M, Freitag F, Reed ML, Stewart WF. Migraine prevalence, disease burden, and the need for preventive therapy. Neurology 2007; 68:343-349.

2. World Health Organization. The world report. Mental health: New understanding, new hope. World Health Organization, Geneva, 2001.

3. Stokes M, Becker WJ, Lipton RB, Sullivan SD, Wilcox TK, Wells L, Manack A, Proskorovsky I, Gladstone J, Buse DC, Varon SF, Goadsby PJ, Blumenfeld AM. Cost of health care among patients with chronic and episodic migraine in Canada and the USA: Results from the International Burden of Migraine Study (IBMS). Headache 2011; 51:1058-1077.

4. Bloudek LM, Stokes M, Buse DC, Wilcox TK, Lipton RB, Goadsby PJ, Varon SF, Blumenfeld AM, Katsarava Z, Pascual J, Lanteri-Minet M, Cortelli P, Martelletti P. Cost of healthcare for patients with migraine in five European countries: Results from the International Burden of Migraine Study (IBMS). J Headache Pain
2012; 13:361-378.

5. Lipton RB, Liberman JN, Kolodner KB, igal ME, Dowson A, Stewart WF. Migraine headache disability and health-related quality-of-life: A population-based case-control study from England. Cephalalgia 2003; 23:441-450.

6. Rybicka $M$, I. BB, Dyluk B, Nowacki P, Chlubek $D$. The role of magnesium in migraine pathogenesis. Potential use of magnesium compounds in prevention and treatment of migraine headaches. J Elementol 2012; 17:345-356.

7. Welch KM, Ramadan NM. Mitochondria, magnesium and migraine. J Nneurol Sci 1995; 134:9-14.

8. Cui Y, Kataoka Y, Watanabe Y. Role of cortical spreading depression in the pathophysiology of migraine. Neurosci Bull 2014; 30:812-822.

9. Weglicki WB, Phillips TM. Pathobiology of magnesium deficiency: A cytokine/ neurogenic inflammation hypothesis. Am J Physiol 1992; 263:R734-R737.

10. Schurks M, Buring JE, Kurth T. Migraine, migraine features, and cardiovascular disease. Headache 2010; 50:1031-1040.

11. Uncini A, Lodi R, Di Muzio A, Silvestri G, Servidei S, Lugaresi A, lotti S, Zaniol P, Barbiroli B. Abnormal brain and muscle energy metabolism shown by ${ }_{3} \mathrm{P}-\mathrm{MRS}$ in familial hemiplegic migraine.] Neurol Scs 1995; 129:214-222.

12. Bigal ME, Bordini CA, Tepper SJ, Speciali JG.. Intravenous magnesium sulphate in the acute treatment of migraine without aura and migraine with aura. A randomized, double-blind, placebo-controlled study. Cephalalgia 2002; 22:345353.

13. Demirkaya S, Vural O, Dora B, TopçuoDlu MA. Efficacy of intravenous magnesium sulfate in the treatment of acute migraine attacks. Headache 2001; 41:171-177.

14. Peikert A, Wilimzig C, Kohne-Volland R. Prophylaxis of migraine with oral magnesium: Results from a prospective, multi-center, placebo-controlled and double-blind randomized study. Cephalalgia 1996; 16:257-263. 
15. Corbo J, Esses D, Bijur PE, lannaccone $\mathrm{R}$, Gallagher EJ. Randomized clinical trial of intravenous magnesium sulfate as an adjunctive medication for emergency department treatment of migraine headache. Ann Emerg Med 2001; 38:621627.

16. Frank LR, Olson CM, Shuler KB, Gharib SF. Intravenous magnesium for acute benign headache in the emergency department: A randomized double-blind placebo-controlled trial. CJEM 2004; 6:327-332.

17. Maizels M, Blumenfeld A, Burchette R. A combination of riboflavin, magnesium, and feverfew for migraine prophylaxis: A randomized trial. Headache 2004; 44:885-890.

18. Choi H, Parmar N. The use of intravenous magnesium sulphate for acute migraine: Meta-analysis of randomized controlled trials. Eur J Emerg Med 2014; 21:2-9.

19. Moher D, Liberati A, Tetzlaff J, Altman DG; PRISMA Group. Preferred reporting items for systematic reviews and metaanalyses: The PRISMA statement. Int J Surg 2010; 8:336-341.

20. Higgins JPT, Green S. Cochrane Handbook for Systematic Reviews of Interventions Version 5.1.0. The Cochrane Collaboration, 2011

21. Borenstein M, Hedges LV, Higgins JPT. Introduction to Meta-Analysis. John Wiley \& Sons, United Kingdom, 2009.

22. Higgins JP, Thompson SG, Deeks JJ, Altman DG. Measuring inconsistency in meta-analyses. BMJ 2003; 327:557-560.

23. Lipsey M, Wilson D (eds). Practical Meta Analysis. Sage, Thousand Oaks, 2001.

24. Egger M, Davey Smith G, Schneider M, Minder C. Bias in meta-analysis detected by a simple, graphical test. BMJ 1997; 315:629-634.

25. Song F, Khan KS, Dinnes J, Sutton AJ. Asymmetric funnel plots and publication bias in meta-analyses of diagnostic accuracy. Int J Epidemiol 2002; 31:88-95.

26. DerSimonian R, Laird N. Meta-analysis in clinical trials. Control Clin Trials 1986; 7:177-188.

27. Pfaffenrath V, Wessely P, Meyer C, Isler HR, Evers S, Grotemeyer KH, Taneri Z, Soyka D, Göbel H, Fischer M. Magnesium in the prophylaxis of migraine - A double-blind, placebo-controlled study. Cephalalgia 1996; 16:436-440.

28. Ginder S, Oatman B, Pollack M. A prospective study of i.v. magnesium and i.v. prochlorperazine in the treatment of headaches. J Emerg Med 2000; 18:311315 .

29. Cete Y, Dora B, Ertan C, Ozdemir C Oktay C. A randomized prospective placebo-controlled study of intravenous magnesium sulphate vs. metoclopramide in the management of acute migraine attacks in the emergency department. Cephalalgia 2005; 25:199-204.

30. Li GS. [Clinical effects of Ozagrel combined with magnesium for treating 120 individuals with migraine]. China Health Industry 2013; 2:71.

31. Shahrami A, Assarzadegan F, Hatamabadi HR, Asgarzadeh M, Sarehbandi $\mathrm{B}$, Asgarzadeh S. Comparison of therapeutic effects of magnesium sulfate vs. dexamethasone/metoclopramide on alleviating acute migraine headache. J Emerg Med 2015;48: 69-76.

32. Liu RY. [Clinical effects of ozagrel combined with magnesium for treating migraine]. China Med Engineering 2013; 21:93.

33. Tang L, Zhou YP. [Clinical study of ozagrel combined with magnesium for treating migraine]. J Prac Med 2011; 27:2531.

34. Wang Q. [Clinical effects of ozagrel combined with magnesium for treating migraine]. China Med Engineering 2013; 21:89.

35. Wang YH, Yu YF. [Clinical study of intravenous magnesium for treating $\mathrm{mi}$ graine]. Journal of Qiqihar Medical College 2010; 31:860.

36. Xu L, Xu Y. [Observation of the effect of intravenous magnesium combined with lidocaine for treating migraine]. Med Infor 2010; 23:2103-2103.

37. Lai Y], Chen RJ. [Effects observation of magnesium sulfate combined with zhengtianwan in migraine]. Chin J Clini Rational Drug Use 2012; 5:20-21.

38. Wang JX. [Observation of effects of magnesium propylvalerate on treating migraine]. Med Industry Infor 2005; 2:115116.

39. Wang J. [Sodium bicarbonate combined with magnesium on treating migraine in 60 cases]. Hebei Med J 1992; 47:1058.

40. Zhang ZL, Sun JZ, Jiang H. [Clinical observation of magnesium on treating migraine in 77 cases]. J Clini Neurol 1991; 4:109.

41. Wang CL. [Observation of magnesium propylvalerate combined with flunarizin on treating migraine in 46 cases]. China
Health Industry 2012; 27:97.

42. Zhou Y, Gao L. [Flunarizine combined with magnesium polarization liquid on treating refractory migraine]. Clinical Focus 1998; 13:664.

43. Hu CS, Zhou LB. [Observation of clinical effects of magnesium valproate combined with venlafaxine $\mathrm{HCl}$ in 54 individual with migraine]. China Modern Mede 2011; 18:62.

44. Koseoglu E, Talaslioglu A, Gonul AS, Kula M. The effects of magnesium prophylaxis in migraine without aura. Magnes Res 2008; 21:101-108.

45. Tarighat Esfanjani A, Mahdavi R, Ebrahimi Mameghani M, Talebi M, Nikniaz $Z$, Safaiyan A. The effects of magnesium, L-carnitine, and concurrent magnesium-L-carnitine supplementation in migraine prophylaxis. Biol Trace Elem Res 2012; 150:42-48.

46. Wang SS, Shan H. [Effects of magnesium sulphate, ergotamine, and flunarizine hydrochloride on treating $\mathrm{mi}-$ graine]. Henan J Prevention Med 2001; 12:366-367.

47. Yang XS, Yang JD, Yuan RF, Xu XP, Zhang LF. [Randomized double blind controlled trial of magnesium valproate in migraine prophylaxis]. China J Hospital Pharmacy 2005; 25:649-651.

48. Bian X, Zhu Y, Xia J, Ai HJ, Guo QY, Lu LB, Zhang QW. [Clinical observation om potassium magnesium asparate oral soluation combined with flinarizine capsule for migraine prophylaxis]. J Comm Med 2013; 11:6-7.

49. Lan QB, Yang C. [Clinical observation of magnesium propylvalerate on treating migraine]. J Youjiang Medical College for Nationalitis 1998; 21:639-640.

50. Tang $X Y$, Lin $W Z$, Zheng $X M$, Liu CS. [Observation of effects of nimodipine and magnesium sulfate combination for treating migraine]. J Qiqihar Medical College 1998; 19:5-6.

51. Tarighat Esfanjani A, Mahdavi R, Ebrahimi Mameghani M, Talebi M, Nikniaz $Z$, Safaiyan A. The effects of magnesium, L-carnitine, and concurrent magnesium-L-carnitine supplementation in migraine prophylaxis. Biol Trace Elem Res 2012; 150:42-48.

52. Taylor FR, Kaniecki RG. Symptomatic treatment of migraine: When to use NSAIDs, triptans, or opiates. Curr Treat OptionsNeurol 2011; 13:15-27.

53. Silberstein SD. Practice parameter: Evidence-based guidelines for migraine headache (an evidence-based review): Report of the Quality Standards Sub- 
committee of the American Academy of Neurology. Neurology 2000; 55:754-762.

54. Stewart WF, Lipton RB, Celentano DD, Reed ML. Prevalence of migraine headache in the United States. Relation to age, income, race, and other sociodemographic factors. JAMA1992; 267:64 69.
55. Steiner TJ, Scher AI, Stewart WF, Kolodner K, Liberman J, Lipton RB. The prevalence and disability burden of adult migraine in England and their relationships to age, gender and ethnicity. Cephalalgia 2003; 23:519-527.
56. Chiu HY, Tsai PS, Lee CC, Liu YT, Huang $\mathrm{HC}$, Chen PY. The association between use of dietary supplements and headache or migraine complaints. Headache 2014; 54:355-363. 